\title{
Current Status of the Laser Diode Array Projector Technology
}

\author{
D. Brett Beasley and Daniel A. Saylor, \\ Optical Sciences Corporation, \\ P.O. Box 8291, \\ Huntsville, AL 35808
}

\begin{abstract}
This paper describes recent developments and the current status of the Laser Diode Array Projector (LDAP) Technology. The LDAP is a state-of-the-art dynamic infrared scene projector system capable of generating high resolution in-band infrared imagery at high frame rates. Three LDAPs are now operational at the US Army Aviation and Missile Command's (AMCOM) Missile Research, Development, and Engineering Center (MRDEC). These projectors have been used to support multiple Hardware-in-the-Loop test entries of various seeker configurations. Seeker configurations tested include an InSb 256x256 focal-plane array (FPA), an InSb 512x512 FPA, a PtSi 640x480 FPA, a PtSi 256x256 FPA, an uncooled 320x240 microbolometer FPA, and two dual field-of-view (FOV) seekers. Several improvements in the projector technology have been made since we last reported in 1997. The format size has been increased to 544x544, and 672x512, and it has been proven that the LDAP can be synchronized without a signal from the unit-under test (UUT). The control software has been enhanced to provide "point and click" control for setup, calibration, image display, image capture, and data analysis. In addition, the first long-wave infrared (LWIR) LDAP is now operational, as well as a dual field of view LDAP which can change its FOV within 0.25 seconds. The projector is interfaced to a Silicon Graphics scene generation computer which is capable of real-time 3-D scene generation. Sample images generated with the projector and captured by an InSb FPA sensor are included in the text.
\end{abstract}

Keywords: Infrared, Scene Projection, Diode lasers, Simulation, FPA testing, Hardware-in-the-loop.

\subsection{INTRODUCTION}

Development of the first imaging Laser Diode Array Projector (LDAP) began in 1993 and was delivered to AMCOM in early 1995. Since that time the Optical Sciences Corporation/Boeing Aerospace/AMCOM team has made significant improvements in the performance and operational capabilities of the technology. During the last year we have transitioned from a single projector to multiple projectors customized for particular seeker testing. Three complete LDAP systems have been fabricated and are used in three separate infrared HWIL test facilities at AMCOM. One of these three has multiple optical configurations to support two seekers with dual-FOV capability. In addition to these three LDAPs there is another LDAP in fabrication at this time, and additional upgrades to the LDAPs are planned for the near future. This paper details some of the advances in the technology which have been made in the past year and characterizes the three operational LDAP systems. In addition, planned upgrades are briefly discussed.

The LDAP is a state-of-the-art dynamic infrared projector system capable of generating high resolution in-band infrared imagery at high frame rates. The system consists of a linear array of custom $\mathrm{Pb}$-salt diode lasers coupled with a high-speed optical scanning system and drive electronics. It is designed specifically for testing sensors which utilize a focal plane array

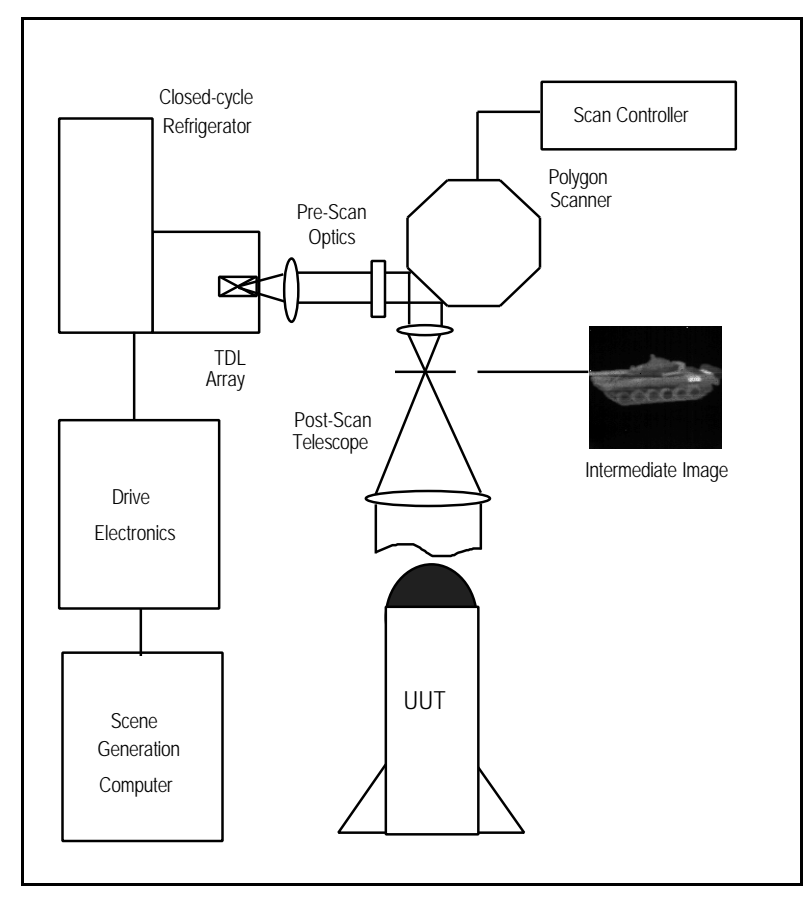

Figure 1. Projector schematic 
(FPA), but can be modified to work with any sensor which has detector integration. Like other scanning projector systems, the LDAP projector takes advantage of the FPA's integration mechanism. The output intensity of each diode laser is temporally modulated in synchronization with the scanning mirror to effectively "paint" a two-dimensional scene across the UUT's field of view (FOV). As the image of the laser array is scanned across the FPA, the appropriate amount of energy is deposited onto each detector to generate the simulated scene. The projector utilizes a "push-broom" scan pattern with a N:1 field interlace (where $\mathrm{N}$ may be set to any desired value such as 2,4,8,or 16) to increase the number of rows in the scene. The projector must scan over each FPA detector at least once during its minimum integration time. In addition, the projector scan rate must be set such that each detector is "painted" the same number of times during each FPA integration cycle. The synchronization is designed such that the UUT may change its integration time or gains at will without affecting the projector's calibration or performance. Figure 1 is a schematic of the projector hardware, and Figure

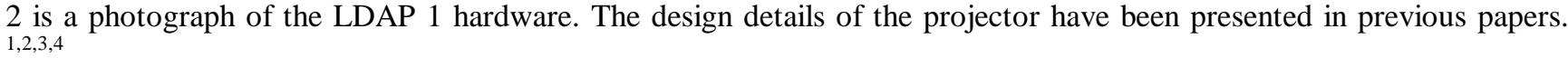

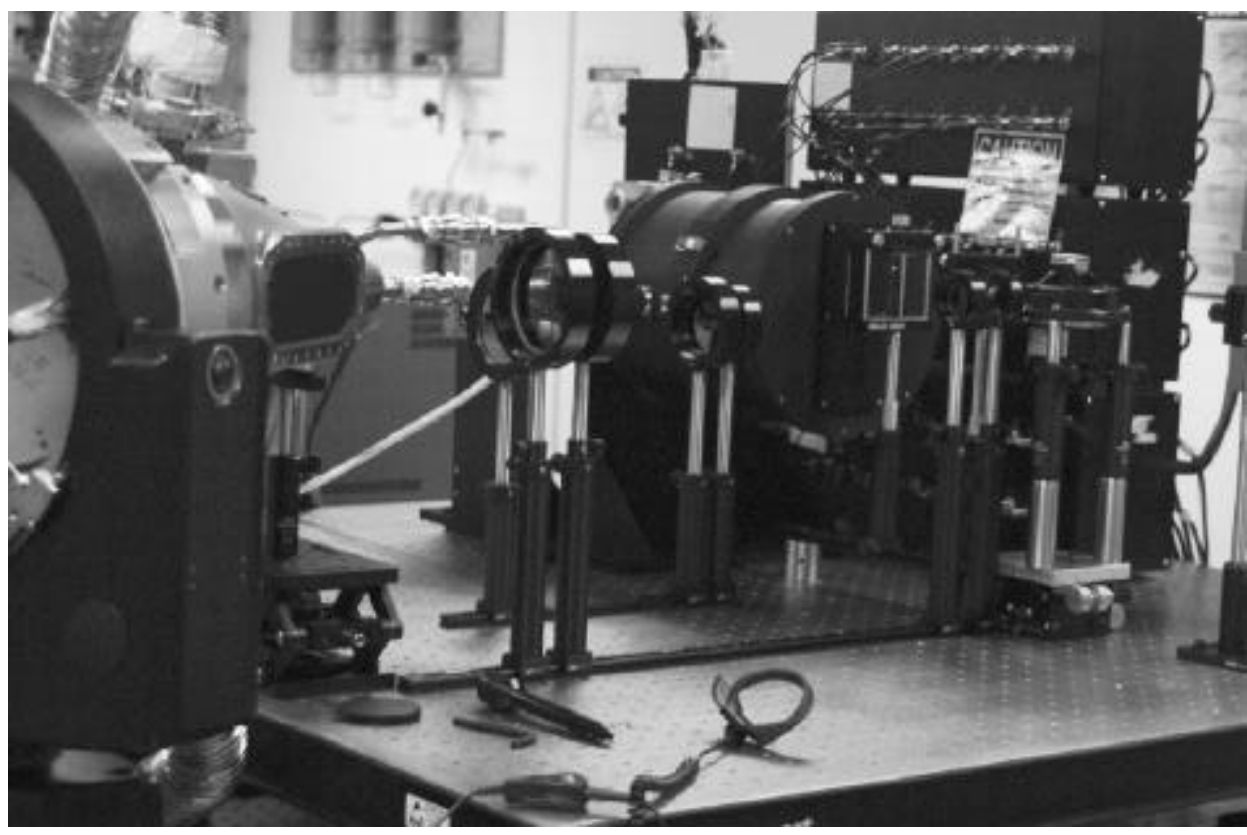

Figure 2. LDAP 1

\subsection{NEW DEVELOPMENTS}

\subsection{Increased Format}

The most significant improvement in the projector performance parameters is the scene format or resolution. The format has increased to $544 \times 544$ for LDAP 1 and $672 \times 512$ for LDAPs 2 and 3. LDAP 1 can also be operated in 256x256 mode at an increased frame rate, if desired. The resolution in the scan direction was increased by changing the update rate of the lasers to increase the number of pixels projected in the scan direction. The resolution in the cross-scan direction could have been achieved by an increase in either the number of lasers or the interlace. The low-cost approach of increasing the interlace by redesigning the polygon mirror was chosen. The 544 vertical resolution was achieved by increasing the number of interlaces to 8:1 and adding four lasers. Due to practical limitations in the construction of the polygon mirrors, a tradeoff of frame rate occurs with this increase. The increased format also required improved optical systems which have a resolution equal to, or better than, the increased format. The pre-scan and post-scan optical systems were custom designed and manufactured to meet the improved resolution requirements.

The increased format necessitates a reduced projector frame rate and increased update rate of the lasers. In order to maintain the same maximum simulated temperature (without adding lasers), the polygon facet size and polygon diameter must remain the same. This entails that the scanner speed cannot be increased without a major redesign. Because the 
polygon must remain the same size and speed, the effective frame rate of the projector is reduced by a factor of two when the interlace is doubled. Thus, the increased interlace reduces the frame rate from $4 \mathrm{kHz}$ to $2 \mathrm{kHz}$. This frame rate, however, is still an order of magnitude larger than that currently available through any other technology. The $2 \mathrm{kHz}$ frame rate is sufficient to support seeker integration times as low as $0.5 \mathrm{msec}$, which is sufficient to support our seekers of interest. The increased resolution in the scan direction is achieved by increasing the digital-to-analog converter (DAC) update rate. The DAC update rate increased from $4.1 \mathrm{MHz}$ to $8.7 \mathrm{MHz}$, which is still within the current $10 \mathrm{MHz}$ capability.

\subsection{Software Enhancements}

The initial prototype projector was operated through a series of DOS-based programs. Each unique task related to laser operation, system calibration, and data collection was executed separately through a text-based command line call. As the hardware and software capabilities increased, operators were required to remember a growing number of program names. As additional projectors were developed, a coordinated approach to the software development became necessary. With the implementation of complete commonality between all LDAP communication hardware, a common software interface for all LDAP systems became possible. The software system currently under development is a "dialog-based" Windows 95 platform application written within the Visual C++ environment. Using the familiar "point and click" navigation technique, the user has complete control over laser operation, image/movie loading, image capture, data analysis, calibration, alignment and other required functions for daily operation. Figure 3 shows a representative screen which is displayed during operation of the LDAP control software. Table 1 shows the menu tree and provides a top-level description of each function.

\begin{tabular}{|c|c|c|}
\hline $\begin{array}{l}\text { Main Menu } \\
\text { Selections }\end{array}$ & $\begin{array}{l}\text { Sub-Menu } \\
\text { Selections }\end{array}$ & Description \\
\hline Setup & ------ & Configuration of communications, electronics. \\
\hline \multirow[t]{5}{*}{ Utilities } & DC3 & Manual operation of individual lasers. \\
\hline & Align & Pattern display and image grab tools for precision alignment. \\
\hline & PCI-VME & Direct read/write to any VME address via Bit 3 PCI card. \\
\hline & Map & LDAP scene to FPA map for input to gain/offset calculation. \\
\hline & Camera & Operation of available UUT/calibration camera functions. \\
\hline \multirow[t]{3}{*}{ Images } & Static & Uploading and display of static patterns and images. \\
\hline & Dynamic & Uploading and display of dynamic images (movies). \\
\hline & Grab & Image capture, display and analysis. \\
\hline \multirow[t]{4}{*}{ Tables } & Initialize & Initialization of gain, offset, linearization, and image memory tables. \\
\hline & Ramp & Loading of default linearization table using full current scale. \\
\hline & Review & Graphical display of linearization, gain, and offset table info. \\
\hline & File Upload & Loading of collected linearization, gain, and offset tables. \\
\hline Calibrate & ----- & $\begin{array}{l}\text { Collection of all necessary information for determination of linearization, } \\
\text { gain, and offset tables. }\end{array}$ \\
\hline
\end{tabular}

Table 1. LDAP Software Menu Tree 


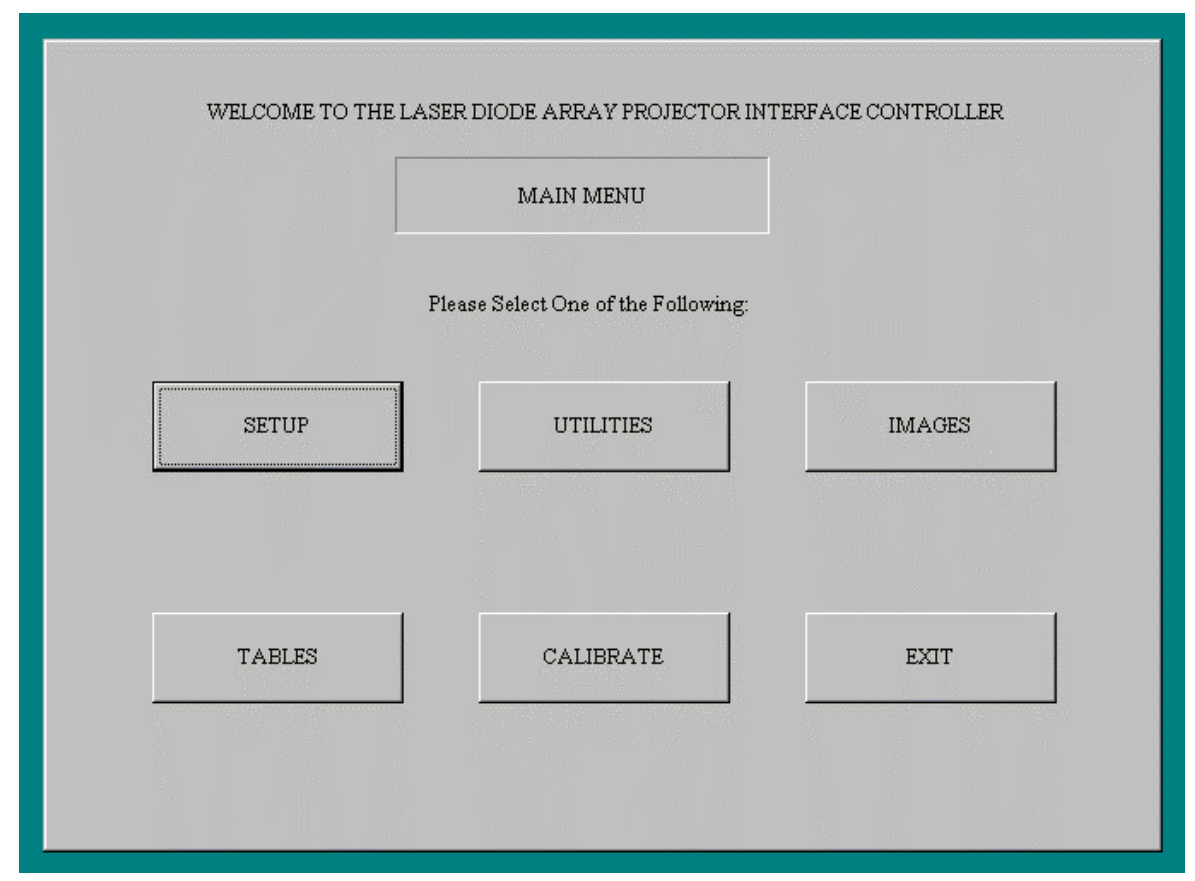

Figure 3. Sample LDAP Software Menu

\subsection{Synchronization}

With a scanning system there is always the issue of synchronizing the projector with the UUT. The LDAP projector must be synchronized such that each detector in the UUT FPA is addressed the same number of times within its integration time. The calibration sensor FPA used with LDAP1 was a 256x256 InSb with rolling integration. Even with the rolling integration, we originally demonstrated that we could synchronize the projector with the FPA with one signal corresponding to the beginning of the FPA integration. The polygon scanner was allowed to operate asynchronously and its frequency was not critical, as long as it was sufficient to complete a full frame in less than the FPA minimum integration time.

During planning for the testing of actual missile seekers, the issue of access to the FPA integration clock (or a derivative) arose. Some seeker systems may not have such a clock available for external access. Thus, the need for synchronization without a signal from the UUT was addressed. The solution turned out to be quite simple. All of the seekers previously tested, and those scheduled to be tested, have a master clock from which the integration clock is derived. Each seeker has a minimum integration time, and all other integration states (or gain states) are an integer multiple of this minimum integration time. Thus, the requirement is to simply set the LDAP scan frequency to match the minimum integration time. For example, the LDAP2 polygon scan frequency is set such that exactly 8 rows are scanned during one UUT integration period. Setting this frequency to exactly match the integration frequency was possible because the scan frequency is tightly controlled with a PLL circuit and the UUT clocks are very stable. Phase and minor phase drift between the two frequencies is not a factor. The frequency is coarsely set with apriori knowledge of the seeker's minimum integration time, and it is fine tuned while monitoring the output of the FPA. A frequency which is too high results in a bright vertical line which rolls through the scene, and a frequency which is too low results in a dark line which rolls through the scene. We have demonstrated this technique with a rolling integration InSb FPA, a commercially available snapshot integration InSb, and two seekers which use snapshot integration InSb FPAs. All currently operational LDAPs use this synchronization technique.

For FPA types which integrate for almost $100 \%$ of the frame period, synchronization is not as critical. The error due to unsynchronized operation is small because the projector frame rate is significantly higher than the UUT frame rate. However, the projector scan frequency is set to be a multiple of the FPA readout frequency. By doing this, the small errors 
which would result from unsynchronized operation are effectively reduced to zero. We have demonstrated this with a PtSi FPA seeker and an uncooled microbolometer FPA sensor.

\subsection{Dual-FOV Systems}

Two of the seekers being tested at AMCOM HWIL facilities are dual field-of-view systems. Each of the seekers has two optical systems with different focal lengths which can be switched at high speed. An LDAP which had the required resolution at both FOVs was required. The solution was a two telescope LDAP in which the FOV could be changed at a higher speed than the UUT could change its FOV. The resulting system is shown in Figure 5. The LDAP has two telescopes mounted on a linear stage. A signal line from the UUT indicates which FOV the seeker is using. Based upon this logic level, the motorized stage positions the correct telescope in the optical path. The stage is designed to change the telescope in 0.25 seconds, which is twice as fast as the UUT switch speed.

\subsection{Long-Wave Infrared Projector}

One of the new LDAP developments this year was a long-wave (LW) IR projector system. The LW LDAP was designed to test two LWIR seekers. One of the seekers utilizes an uncooled microbolometer FPA and the other has a HgCdTe FPA. The HgCdTe FPA was straightforward because of its similarity to the InSb FPA operation. The microbolometer array seeker, however, presented new challenges due to the difference in the physics of the detectors. The microbolometer array does not integrate photons and store charge until it is read out. Rather the detector element changes resistance in relation to its temperature which is a result of amount of energy incident upon it. The readout electronics sample the elements'

resistance at the frame rate of the FPA. The LDAP frame rate had to be sufficient to insure that the average temperature of the microbolometer element was correct and that there was not excessive ripple in the detector output due to the duty factor of the laser addressing the element. It was proven that the LDAP could project a realistic scene with no scanning or sampling effects in the microbolometer FPA. The LDAP's high frame rate combined with setting the scan frequency to a multiple of the FPA frame rate resulted in scene with no flicker effects.

One challenge to the LW projector, however, was achieving a sufficient maximum apparent temperature. Because of the wide spectral bandwidth of the microbolometer array, and the characteristic of Planck's law, a much larger amount of laser power is required to simulate a given temperature. Thus, it was necessary to improve the collection efficiency of the LDAP optical system. This was achieved by using a much larger diameter polygon mirror because the polygon facet is the aperture stop of the system. The larger polygon, however, resulted in a lower frame rate. This trade-off was acceptable because of the $100 \%$ integration time of the microbolometer array and its relatively slow $30 \mathrm{~Hz}$ frame rate.

\subsection{Rectangular IFOV}

The design of the LDAP has proven to be flexible enough to support special needs without hardware modification. One of these needs was the capability to test a seeker with a rectangular, instead of square, IFOV using an LDAP designed for square IFOVs. This need was supported through the LDAP's capability to set the number of pixels in the scan direction through software. The resolution in the scan direction is set by the phase lock loop circuit's "divide-by" factor. This parameter can be set via software allowing any resolution up to 1024 in the scan direction. Thus, any desired aspect ratio can be generated. The optical FOV of the projector is then set to match the FOV of the seeker in the scan direction and the LDAP resolution then matches the UUT.

\subsection{OPERATIONAL PROJECTORS}

Three LDAPs are now operational at the US Army AMCOM Missile Research, Development, and Engineering Center. These projectors have been used to support multiple Hardware-in-the-Loop test entries of various seeker configurations. Seeker configurations tested include an InSb 256x256 FPA, an InSb 512x512 FPA, a PtSi 640x480 FPA, a PtSi 256x256 FPA, and an uncooled 320x240 microbolometer FPA. Two of these seekers have dual-FOV optical systems which may be switched during the simulation. Details of the three operational LDAPs are discussed in the following sections. 


\subsection{LDAP 1}

LDAP 1 is a $544 \times 544$ mid-wave infrared projector. It was designed for the Imaging IR Simulator System2 (IIRSS2) HWIL facility. Table 2 contains the specifications for the projector. Figure 4 is a sample image projected by LDAP 1 in 256x256 mode and captured using an Amber 256x256 InSb FPA. All sample images were imported into this paper using Microsoft ${ }^{\circledR}$ Word, and printed on a $600 \mathrm{dpi}$ laserjet printer.

\begin{tabular}{|l|c|}
\hline $\begin{array}{l}\text { Performance } \\
\text { Parameter }\end{array}$ & Value \\
\hline \hline Spatial Resolution & 256X256 or 544x544 \\
\hline Number of lasers & 68 \\
\hline Field of View & Multiple \\
\hline Emission Wavelength & $4.6 \mu \mathrm{m}$ \\
\hline Field Rate & $16 \mathrm{kHz}$ \\
\hline Frame Rate & $4 \mathrm{kHz}$ or $2 \mathrm{kHz}$ \\
\hline
\end{tabular}

Table 2. LDAP1 Specifications

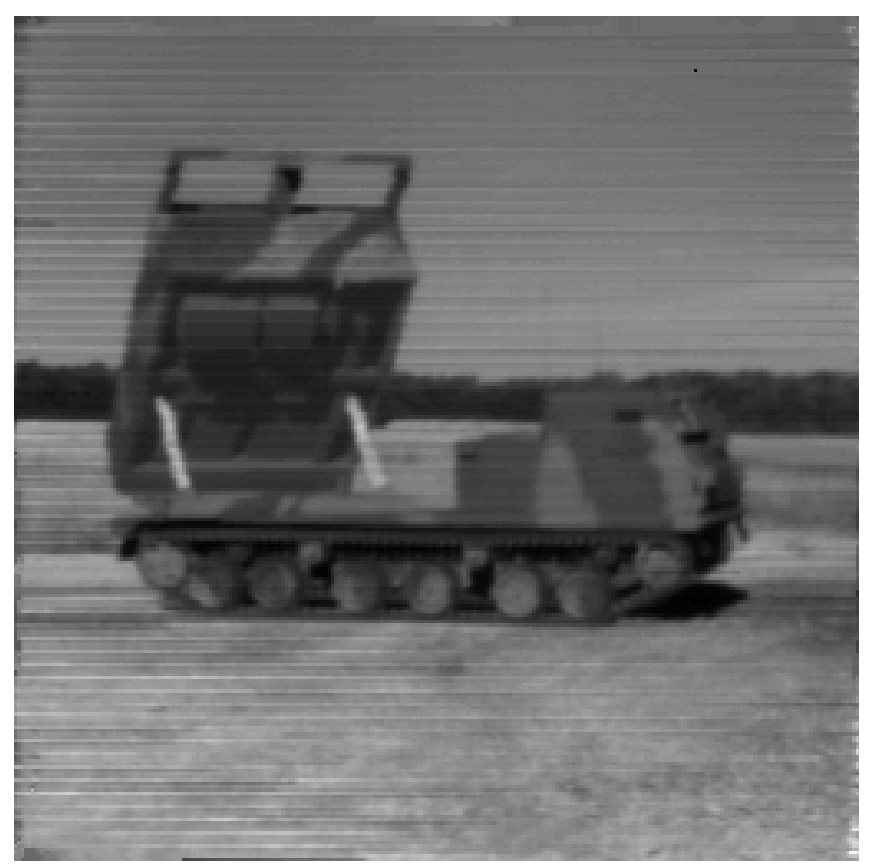

Figure 4 - Projection of Simulated Launcher.

\subsection{LDAP 2}

LDAP 2 was designed and fabricated to support the IIRSS3 HWIL facility. This projector may be operated in either of two configurations to support the testing of two unique seekers. Both of the seekers being tested have dual FOV optical systems, therefore both LDAP configurations have a dual FOV capability. Configuration A of LDAP 2 has a $544 \times 544$ format which is used to test a 512x512 InSb FPA seeker. Configuration B if LDAP 2 has a 672x512 format which is used to test a 640x480 PtSi FPA seeker. Changing the LDAP configuration from 2A to $2 \mathrm{~B}$ requires swapping the complete optical train and polygon scanner. The format size must also be changed via software. Both configurations of LDAP 2 are detailed in the following sections.

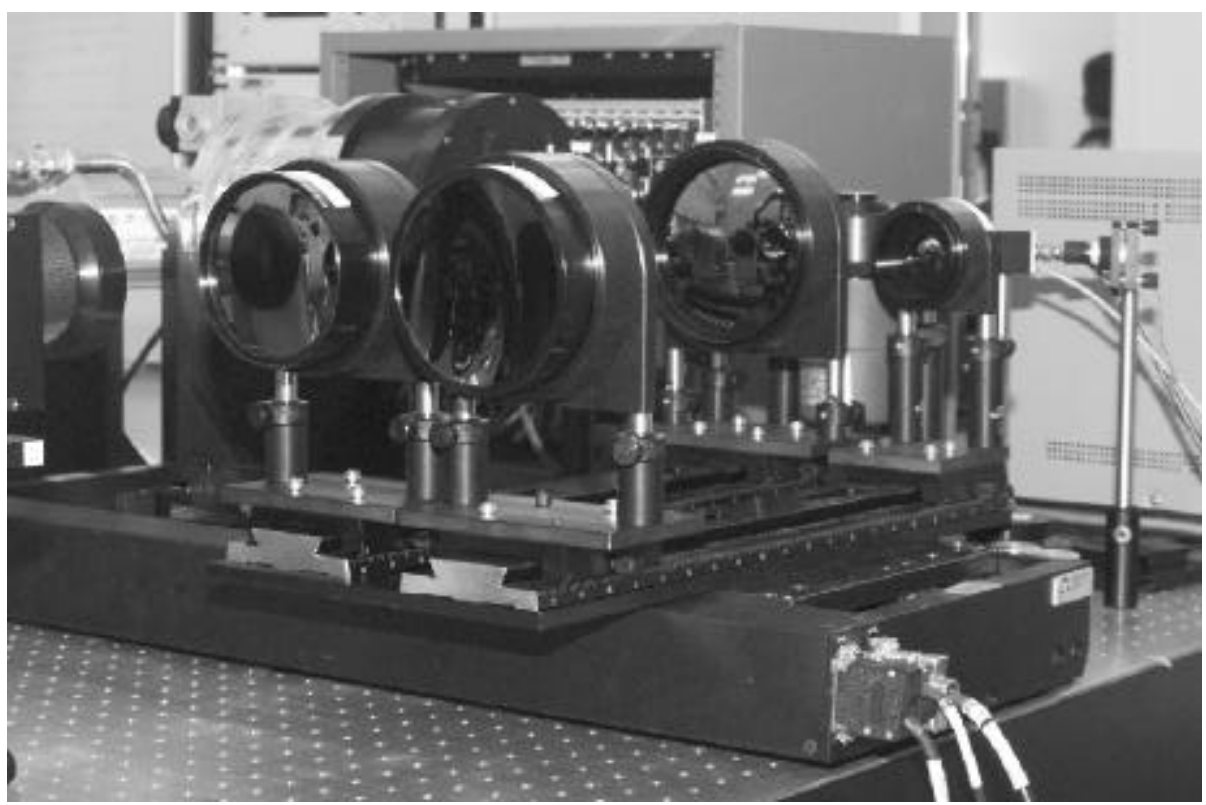

Figure 5. LDAP 2 with FOV Changer 


\subsubsection{LDAP 2A}

Table 3 contains the performance parameters for LDAP 2A. Figure 6 is an image projected using LDAP 2A and collected using a $512 \times 512 \mathrm{InSb}$ FPA camera. Figure 7 is a $512 \times 413$ image projected using LDAP 2A and collected with a 512x512 InSb FPA camera.

\begin{tabular}{|l|c|}
\hline $\begin{array}{l}\text { Performance } \\
\text { Parameter }\end{array}$ & Value \\
\hline \hline Spatial Resolution & $544 \times 544$ \\
\hline Number of lasers & 68 \\
\hline Field of View & Dual $\left(\approx 9^{\circ}\right.$ and $\left.2^{\circ}\right)$ \\
\hline Emission Wavelength & $4.7 \mu \mathrm{m}$ \\
\hline Field Rate & $16 \mathrm{kHz}$ \\
\hline Frame Rate & $2 \mathrm{kHz}$ \\
\hline Max. Apparent Temp. & $\approx 525 \mathrm{~K}$ \\
\hline
\end{tabular}

Table 3. LDAP 2A Specifications

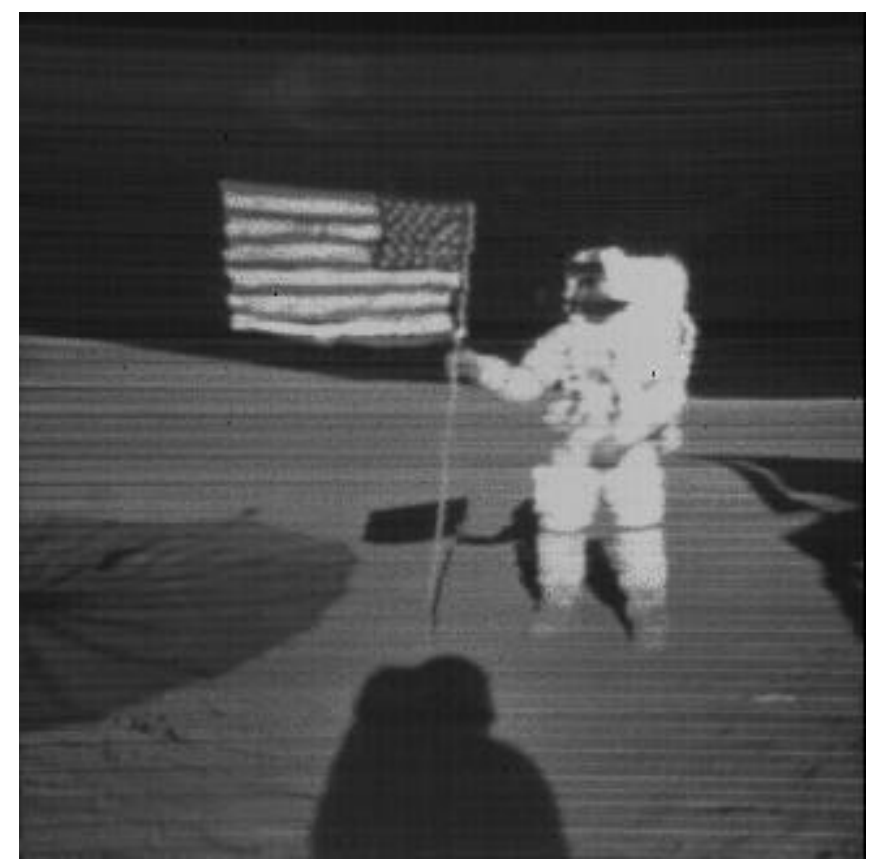

Figure 6. "Apollo" 512x512 Projected Image

\subsubsection{LDAP 2B}

LDAP 2B is a MWIR projector designed to test a seeker with a PtSi FPA and a dual FOV optical system. Table 3 contains the performance parameters for LDAP 2B. Figure 5 is a photograph of the system showing the two telescopes and the FOV changer.

\begin{tabular}{|l|c|}
\hline $\begin{array}{l}\text { Performance } \\
\text { Parameter }\end{array}$ & Value \\
\hline \hline Spatial Resolution & $672 \times 512$ \\
\hline Number of lasers & 64 \\
\hline Field of View & Dual $\left(\approx 12 \times 9^{\circ}\right.$ and $\left.6 \times 4.5^{\circ}\right)$ \\
\hline Emission Wavelength & $4.7 \mu \mathrm{m}$ \\
\hline Field Rate & $16 \mathrm{kHz}$ \\
\hline Frame Rate & $2 \mathrm{kHz}$ \\
\hline Max. Apparent Temp. & $\mathrm{TBD}$ \\
\hline
\end{tabular}

Table 3. LDAP 2B Specifications

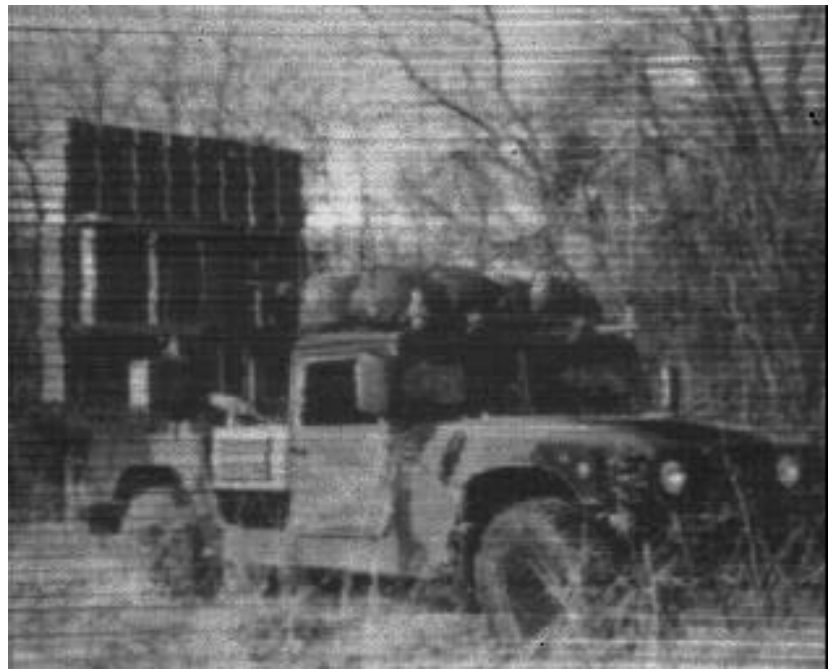

Figure 7. "Launcher" 512x413 Projected Image 


\subsection{LDAP 3 LWIR Projector}

LDAP 3 is a $672 \times 512$ long-wave infrared projector. It was designed for the Millimeter Imaging IR Simulator System (MM/IIRSS) HWIL facility. This facility is designed for testing dual-mode millimeter radar and imaging IR seekers. As discussed earlier in the paper, this projector was designed to test a seeker which utilizes a microbolometer FPA. It has also been used in a $512 \times 512$ configuration to test a seeker with a $\mathrm{HgCdTe}$ FPA. Table 4 contains the specifications for the projector. Figure 8 is a photograph of the system showing the larger polygon scanner.

\begin{tabular}{|l|c|}
\hline $\begin{array}{l}\text { Performance } \\
\text { Parameter }\end{array}$ & Value \\
\hline \hline Spatial Resolution & $672 \times 512$ or $544 \times 544$ \\
\hline Number of lasers & 68 \\
\hline Field of View & Wide or Narrow \\
\hline Emission Wavelength & $8.5 \mu \mathrm{m}$ \\
\hline Field Rate & $4.8 \mathrm{kHz}$ or $16 \mathrm{kHz}$ \\
\hline Frame Rate & $600 \mathrm{~Hz}$ or $2 \mathrm{kHz}$ \\
\hline $\begin{array}{l}\text { Max. Apparent Temp. } \\
\text { (UUT dependent) }\end{array}$ & $370 \mathrm{~K}$ or $>650 \mathrm{~K}$ \\
\hline
\end{tabular}

Table 4. LDAP 3 Specifications

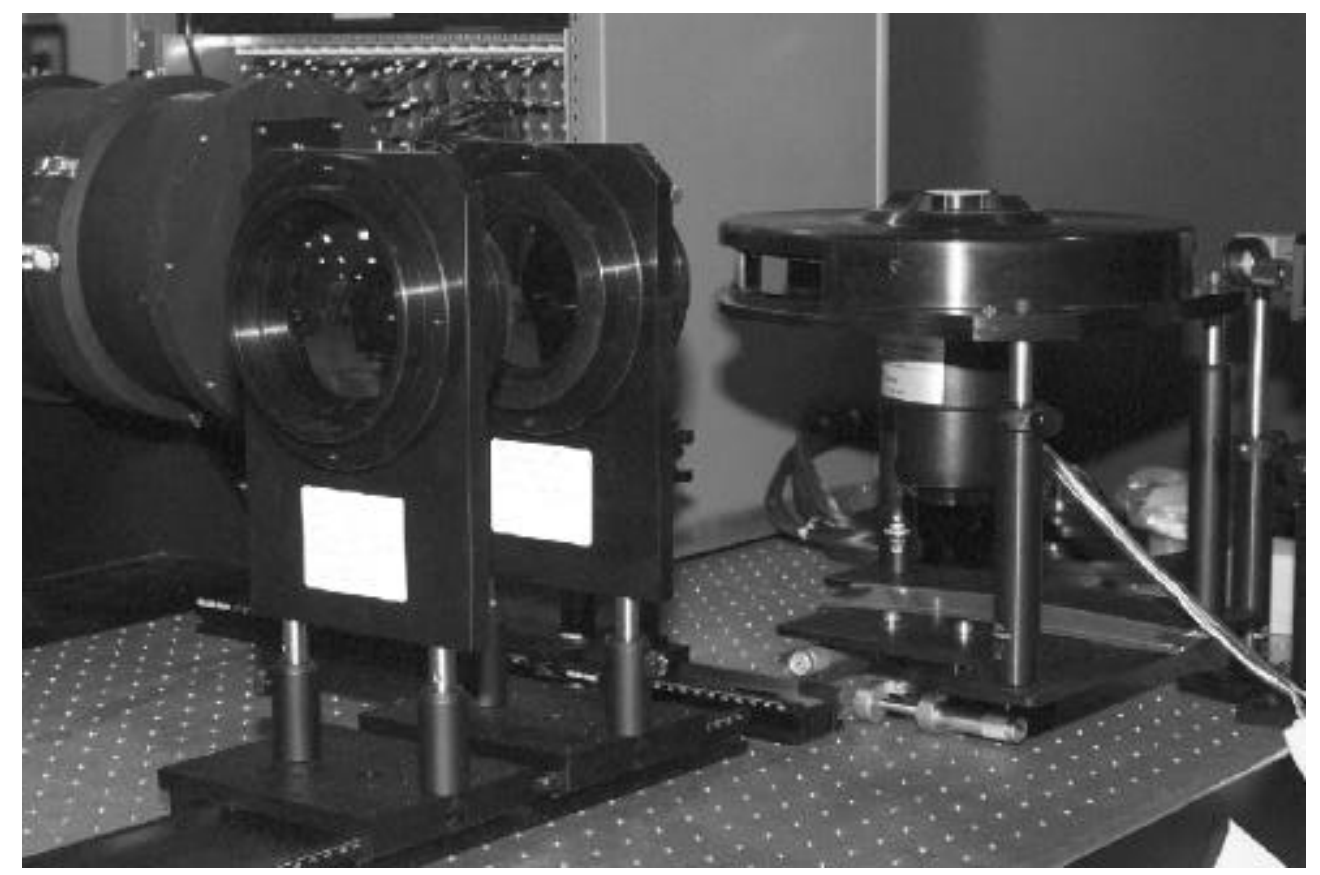

Figure 8. LDAP 3 Long-Wave IR Projector

\subsection{FUTURE DEVELOPMENTS}

\subsection{Implementation of LDAP Modification to Remove Scan Lines}

The current MWIR LDAP systems have achieved uniformity levels of $96-97 \%$ as measured with a mid-scale full-FOV uniform projected source. These levels are comparable to other published projector technology uniformity levels measured in a similar manner. However, unlike the other technologies, the distribution of the non-uniformity within the LDAP scenes is largely arranged in a low/mid frequency pattern in the cross-scan directions. The source of this "scan line" nonuniformity has been identified and preliminary testing has shown that the lines can be reduced or removed through a straightforward re-design. Reduction, or elimination, of this primary source of non-uniformity should greatly increase the 
projected uniformity of the LDAP scenes. All previously built LDAP systems can be modified to incorporate this redesign.

\subsection{Increase format to $1024 \times 1024$}

The LDAP electronics were redesigned in 1996 to accommodate a real-time scene generator interface and to support realtime NUC. Although the projector had a 256x256 format, the electronics were designed at that time to accommodate significant growth in the format. Specifically, the tables within the memory cards used to hold the linearization, gain, offset, and image information were designed to handle a 1024x1024 format. The projector format has been increased by increasing the interlace fields and DAC update rate. With this proven approach, and the built-in potential for a further increase in format, AMCOM plans to increase the interlace to $16: 1$ to provide a $1024 \times 1024$ format. Using this approach the $1024 \times 1024$ format can be attained at a cost of approximately $\$ 15,000$.

\subsection{FMS mountable system}

The current LDAP was designed for fixed mounting on an optical bench within the HWIL laboratory. Because the LDAP is stationary, synthetic line-of-sight (SLOS) techniques must be used in the HWIL simulation. SLOS involves the injection of forcing functions into the seeker's gimbal control loop to generate LOS rates. It is sometimes desirable to use true LOS techniques when accessibility to the seeker's electronics is limited. This requires that the projector move relative to the seeker's LOS to generate a target LOS rate. This is usually achieved by using a five axis flight motion simulator with the projector mounted on the outer gimbal of the FMS. A projector which is to be mounted on a five axis FMS must be designed for reduced weight and capable of withstanding acceleration and vibrations. The LDAP is capable of operating on a FMS, but the mechanical system must be redesigned for reduced weight and increased stiffness.

\subsection{CONCLUSIONS}

The US Army Aviation and Missile Command's laser diode array projector is capable of generating realistic dynamic infrared scenes in real-time. There are now three LDAPs which are operational at the Missile Research, Development, and Engineering Center. These projectors have been used to support multiple Hardware-in-the-Loop test entries of various seeker configurations. The LDAP's format size and image quality is equal to or better than other available technologies. There are plans for future upgrades to increase the format size, improve the spatial non-uniformity, increase the dynamic range, and support other HWIL test objectives.

\subsection{ACKNOWLEDGMENTS}

This work was sponsored by the US Army Aviation and Missile Command's Missile Research, Development, and Engineering Center. The authors would like to thank Mr. Alex Jolly, Mr. Bill Sholes, Mr. Scottie Mobley, and Mr. Jim Buford, all of USAAMCOM, for their support of our efforts.

\subsection{REFERENCES}

1. D.B. Beasley and J.B. Cooper, “Diode Laser Arrays for Dynamic Infrared Scene Projection”, Characterization, Propagation, and Simulation of Sources and Backgrounds III, Wendell R. Watkins, Dieter Clement, Editors, Proc. SPIE 1967, pp. 77-88 (1993).

2. D.B. Beasley, J.B. Cooper, S. Mobley, and J. Buford, "Diode Laser Based Infrared Scene Projector", Targets and Backgrounds: Characterization and Representation, Wendell R. Watkins, Dieter Clement, Editors, Proc. SPIE 2469, pp. 20-29 (1995).

3. J.B. Cooper and D.B. Beasley, "Optical Design of a Diode Laser Based Dynamic IR Scene Projector", Targets and Backgrounds: Characterization and Representation, Wendell R. Watkins, Dieter Clement, Editors, Proc. SPIE 2469, pp. 146 -156 (1995). 
4. D.B. Beasley and J.B. Cooper, "Performance Capabilities and Utilization of MICOM's Diode Laser Based Infrared Scene Projector Technology”, Technologies for Synthetic Environments: Hardware-in-the-Loop Testing, Robert L. Murrer, Jr., Editor, Proc. SPIE 2741, pp. 110-118 (1996). 